Check for updates

Cite this: RSC Adv., 2018, 8, 40471

Received 27th September 2018 Accepted 27th November 2018

DOI: $10.1039 / \mathrm{c} 8 \mathrm{ra0} 8021 \mathrm{f}$

rsc.li/rsc-advances

\section{Converting ginsenosides from stems and leaves of Panax notoginseng by microwave processing and improving their anticoagulant and anticancer activities}

\author{
Yuan Qu, (D) ${ }^{a}$ Hui-Ying Liu, ${ }^{a}$ Xiao-Xi Guo, ${ }^{a}$ Yan Luo, ${ }^{b}$ Cheng-Xiao Wang, (DD a \\ Jiang-Hua He, ${ }^{a}$ Tian-Rui $\mathrm{Xu}^{a}{ }^{a}$ Ye Yang (D) *a and Xiu-Ming Cui ${ }^{\star a}$
}

A microwave processing technology was applied to degrade saponins from the stems and leaves of Panax notoginseng. Six transformation products (1-6), named 20(S)-ginsenoside $\mathrm{Rg}_{3}(1), 20(R)$-ginsenoside $\mathrm{Rg}_{3}$ (2), notoginsenoside $\mathrm{SFt}_{3}$ (3), ginsenoside $\mathrm{Rk}_{1}(4)$, ginsenoside $\mathrm{Rg}_{5}(5)$, and $20(S)$-ginsenoside $\mathrm{Rh}_{2}(6)$ were isolated and identified from a microwave processed extract of the stems and leaves of $P$. notoginseng (MEL). This transformation method was also applied for producing the minor ginsenosides in flowers, seeds and pedicels of $P$. notoginseng. The extract and compounds 1-6 in MEL were evaluated in vitro for anticancer and anticoagulant activities. The results showed that the MEL extract and transformation products had outstanding inhibitory activities against human cervical cancer Hela and lung cancer A549 cells. The strongest inhibitory effect was observed for $20(S)-\mathrm{Rh}_{2}(6)$ with an $I C_{50}$ value of $8.23 \mu \mathrm{M}$ in Hela cells. Moreover, the results showed that the MEL significantly prolonged prothrombin time in a concentration-dependent manner. The anticoagulant effect of the MEL improved with the increased contents of $\mathrm{Rk}_{1}, \mathrm{Rg}_{5}$, and $\mathrm{SFt}_{3}$.

\section{Introduction}

Panax notoginseng (Burk.) F. H. Chen has been widely used as a herbal medicine in China. It was recorded in the Compendium of Materia Medica that the stems and leaves of P. notoginseng (PNL) were traditionally used to treat bone fractures, eliminate swelling, and stop bleeding. The PNL is also used as a functional food in folk medicine, as PNL is rich in nutrients and characterized by high protein, high dietary fiber, and low fat. ${ }^{1}$ Fresh leaves are suitable for eating as a vegetable and dried as a tea. ${ }^{2}$

More than 40 chemical constituents have been isolated from the PNL. The main components are protopanaxadiol type saponins, such as notoginsenosides $\mathrm{Fa}, \mathrm{Fc}$, and $\mathrm{Fe}$, and the ginsenosides $\mathrm{Rb}_{1}, \mathrm{Rc}, \mathrm{Rb}_{2}$, and $\mathrm{Rb}_{3}$ (Fig. 1). ${ }^{3}$ The saponins have gained prominence owing to their potential pharmaceutical values of analgesic, hypolipidemic, anticancer, anti-arrhythmic,

\footnotetext{
${ }^{a}$ Yunnan Provincial Key Laboratory of Panax notoginseng, Key Laboratory of Panax notoginseng Resources Sustainable Development and Utilization of State Administration of Traditional Chinese Medicine, University Based Provincial Key Laboratory of Screening and Utilization of Targeted Drugs, Faculty of Life Science and Technology, Kunming University of Science and Technology, No. 727 South Jingming Rd., Chenggong District, Kunming, 650500, China. E-mail: yangyekm@ 163.com; sanqi37@vip.sina.com; Tel: +86-183-8715-6001; +86-183-8718-6037

${ }^{b}$ College of Materials and Chemical Engineering, Chongqing University of Arts and Science, Chongqing, 402160, China
}

anticoagulatory, anti-inflammatory, and anti-aging activities., ${ }^{\mathbf{4 5}}$ The interesting structures are less-polar saponins losing some glycosyl moieties such as $20(S / R)$-ginsenosides $\mathrm{Rg}_{3}$ and $\mathrm{Rh}_{2}$ because of more effective anticancer activities. ${ }^{6,7}$ The Shenyi capsule containing ginsenoside $\mathrm{Rg}_{3}$ was clinically applied for treating non-small cell lung cancer in China. ${ }^{8}$ The common methods used for producing less-polar saponins are acid-base degradation, ${ }^{9}$ enzymatic degradation, ${ }^{\mathbf{1 0}}$ microbial transformation, ${ }^{11}$ and steam processing. ${ }^{12}$ At present, microwave processing method as a novel "green" solvent extraction technology is developed to increase the content of minor ginsenosides from white ginseng. ${ }^{\mathbf{1 3 , 1 4}}$ This green extraction process is often used for extracting natural products owing to a faster extraction rate, less consumption of organic solvent, and lower costs of sample preparation, ${ }^{\mathbf{1 5}, 16}$ but more rarely in the case of transformation. The heat processing by microwave method for producing the minor ginsenosides gained high yields within about 60 minutes. ${ }^{13}$ The microwave time was much shorter than used in the traditional heating method (over 20 hours). ${ }^{12}$

We applied the microwave processing method to accelerate the transformation of minor ginsenosides from the roots of $P$. notoginseng and determined the optimal conversion conditions using response surface methodology. ${ }^{17}$ The roots of $P$. notoginseng are traditionally used as medicine, but a huge amount of PNL is often considered waste. In this study, the microwave method was applied to effectively obtain a microwave 


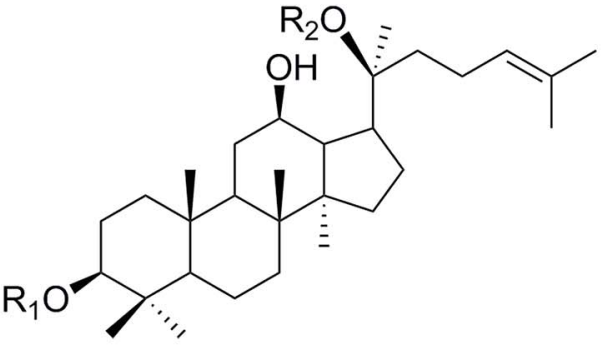

R1

\begin{tabular}{|c|c|c|}
\hline notoginsenoside-Fa & $\mathrm{Glc}(2-I) \mathrm{Glc}(2-I) \mathrm{Xyl}$ & Glc(2-I)Glc \\
\hline ginsenoside- $\mathrm{Rb}_{1}$ & Glc(2-I)Glc & Glc(6-I)Glc \\
\hline ginsenoside-Rc & Glc(2-I)Glc & Glc(6-I)Araf \\
\hline notoginsenoside-Fc & GIc(2-I)G|c2-IXy| & Glc(6-I)Xyl \\
\hline ginsenoside- $R b_{2}$ & $\mathrm{Glc}(2-I) \mathrm{Glc}$ & Glc(6-I)Arap \\
\hline ginsenoside- $\mathrm{Rb}_{3}$ & $\mathrm{Glc}(2-1) \mathrm{Glc}$ & GIc(6-I)Xyl \\
\hline insenoside-l & Glc $(2-I) G \mid c$ & Glc \\
\hline
\end{tabular}

notoginsenoside-Fe Glc

ginsenoside- $\mathrm{Rd}_{2} \quad$ Glc

Glc
R2

Glc(6-I)Araf

GIc(6-I)Arap

$\operatorname{Glc}(6-I) X y \mid$
gypenoside-IX
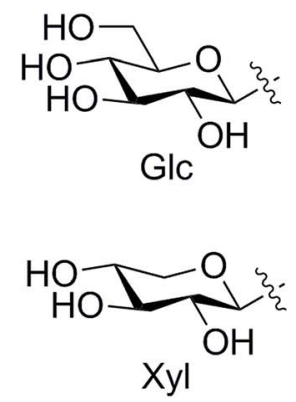<smiles>CC1(O)C(O)COC(O)C(O)C1O</smiles><smiles>OC1C2OC3C(O)C1OC3C2O</smiles>

Araf

Fig. 1 Structures of saponins in the stems and leaves of Panax notoginseng. Glc, D-glucopyranosyl; Xyl, D-xylopyranosyl; Ara(f), L-arabinofuranosyl; Ara(p), L-arabinopyranosyl.

processing extract from stems and leaves of $P$. notoginseng (MEL). Six known compounds (1-6) were isolated from the MEL and identified by nuclear magnetic resonance spectroscopic data (Fig. 2). Moreover, the anticancer and anticoagulant effects of the MEL and the transformation products (1-6) were evaluated during microwave processing in vitro.

\section{Materials and methods}

\subsection{Materials and reagents}

The different parts of $P$. notoginseng (including stems and leaves, flowers, fruits, and pedicels) were purchased from the wenshan market in Yunnan province of China in October 2016. The samples were identified by Dr Xiuming Cui of Kunming University of Science and Technology. The nuclear magnetic resonance spectra (NMR) was recorded by a Bruker Avance III 500 spectrometer (Bruker Corporation, Berlin, Germany). Formic acid (HPLC grade), acetonitrile (HPLC grade), dimethyl sulfoxide (DMSO), 3-[4,5-dimethylthiazol-2-yl]-2,5-diphenyltetrazolium bromide (MTT), penicillin, streptomycin, L-glutamine and methanol were purchased from Sigma-Aldrich (St. Louis., MO, USA). Fetal bovine serum (FBS) and trypsin were purchased from Gibco BRL (Gland Island, NY, USA). All the other reagents and solvents were of analytical grade.

\subsection{Preparation of processed PNL using the microwave processing method}

The dried stems and leaves of $P$. notoginseng (PNL, $50 \mathrm{~g}$ ) were powdered and ultrasonically extracted with $70 \%$ aqueous 


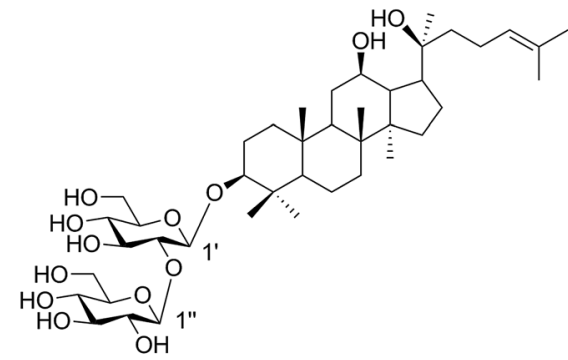

20(S)-ginsenoside- $\mathrm{Rg}_{3}(1)$
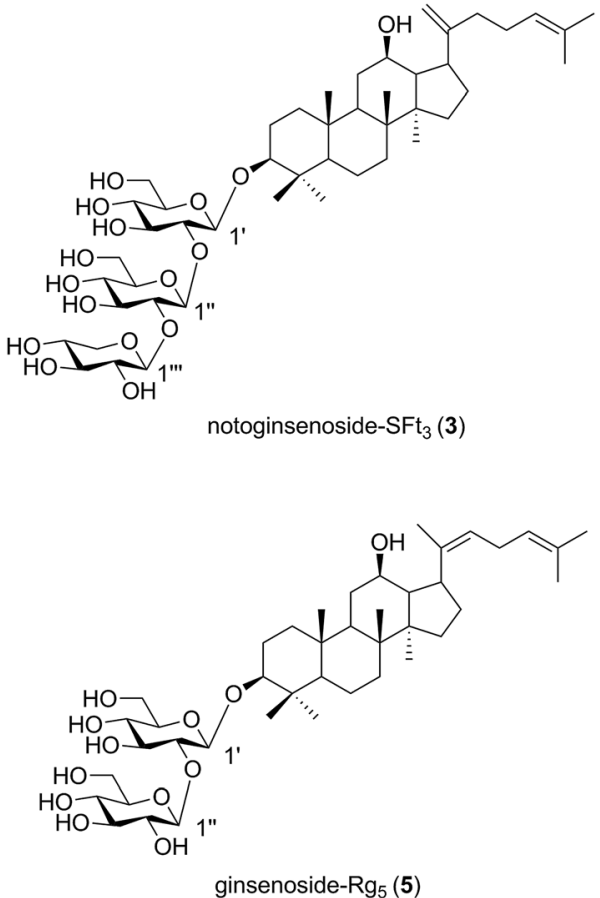
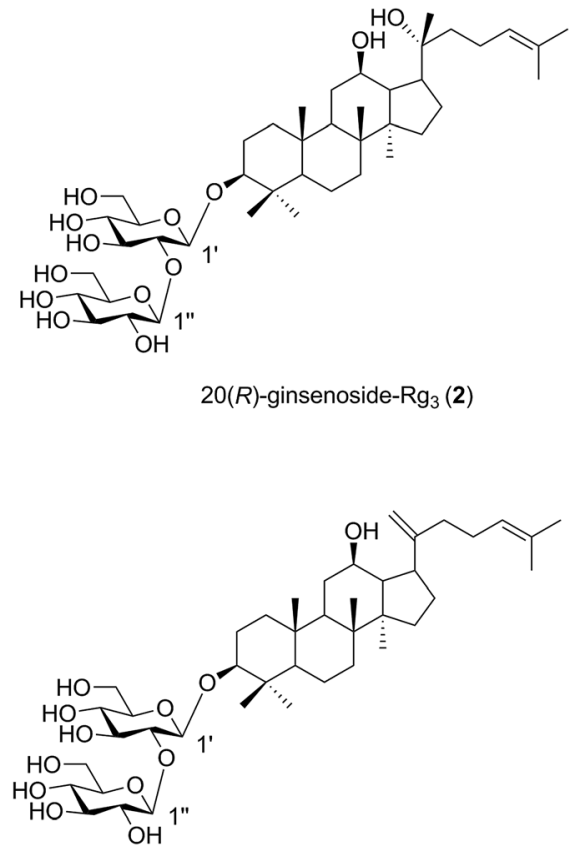

ginsenoside- $\mathrm{Rk}_{1}(\mathbf{4})$

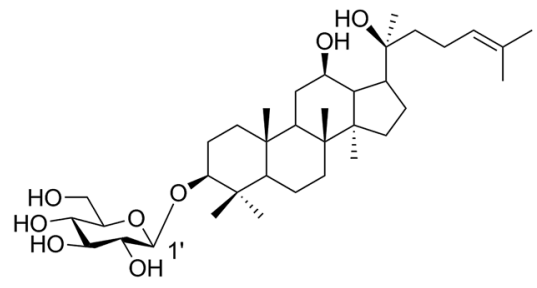

20(S)-ginsenoside- $\mathrm{Rh}_{2}(6)$

Fig. 2 Structures of transformation products 1-6 isolated from microwave processing extract of the stems and leaves of Panax notoginseng.

$\mathrm{MeOH}$, three times for $45 \mathrm{~min}$. After filtration and concentration, the extract of stems and leaves of P. notoginseng (EL, 11.25 g) was obtained with a yield of $22.5 \%$. In the same way, the yields of the extracts of flowers (EF), seeds (ES), and pedicels (EP) were $36.36 \%, 6.48 \%$ and $25.48 \%$, respectively.

The extract samples from different parts of $P$. notoginseng (EL, EF, ES and EP) was treated using microwave processing method as reported previously. ${ }^{17} 160 \mathrm{mg}$ of the extract was added to the water $(10 \mathrm{~mL})$ in a microwave extraction system (model no: MDS-6G) manufactured by Sineo microwave chemical technology Co. Ltd. (Shanghai, China). Considering the influence of microwave temperature, microwave power and time, the temperature ranged from $60{ }^{\circ} \mathrm{C}$ to $180{ }^{\circ} \mathrm{C}$, microwave power ranged from $300 \mathrm{~W}$ to $1000 \mathrm{~W}$, and time ranged from $5 \mathrm{~min}$ to $50 \mathrm{~min}$. After microwave treatment, the sample solution was centrifuged and the supernatant was added to the water for $10 \mathrm{~mL}$. The obtained samples were filtered through a $0.45 \mu \mathrm{m}$ filter membrane for HPLC analysis. The yields of transformation products were obtained from HPLC data.

$$
\text { Yield }(\%)=M_{\mathrm{g}}(\mathrm{g}) / M_{\mathrm{s}}(\mathrm{g})
$$

$M_{\mathrm{g}}$ is the content of transformation products and $M_{\mathrm{s}}$ is total amount of the sample.

\subsection{Identification of MEL from the stems and leaves of $P$. notoginseng during microwave processing method by UHPLC- ESI-Q-TOF-MS}

The EL extract ( $10 \mathrm{~g}$ ) was processed at a temperature of $150{ }^{\circ} \mathrm{C}$ and microwave power of $500 \mathrm{~W}$ for $20 \mathrm{~min}$. After removal of the solvent, microwave processing extract of stems and leaves of $P$. notoginseng (MEL, $9.04 \mathrm{~g}$ ) was obtained with a yield of $90.4 \%$. The composition of MEL was performed on an Agilent 1290 Infinity II LC system (UHPLC) coupled to an Agilent 6530 quadrupole time-of-flight mass spectrometry system (Q-TOF/ MS) (Agilent Technologies, California, America). Samples were separated on an Agilent Poroshell 120 EC-C18 column $(4.6 \mathrm{~mm}$ $\times 75 \mathrm{~mm}, 2.7 \mathrm{im}$ ) at a rate of $0.6 \mathrm{~mL} \mathrm{m^{-1 }}$ using an Agilent 1290 Infinity II LC system. The mobile phases consisted of $0.1 \%$ 
$(\mathrm{v} / \mathrm{v})$ formic acid water $(\mathrm{A})$ and $0.1 \%(\mathrm{v} / \mathrm{v})$ formic acid acetonitrile (B) in the gradient program: 0-10 $\mathrm{min}, 10-25 \%$ (B); 10-50 min, 25-40\% (B); 50-65 min, 40-60\% (B); 65-70 min, 60-100\% (B). For MS detector performed on a Q-TOF/MS, mass spectrometry conditions of electrospray ionization source (ESI) in positive ion detection mode were as follows: capillary voltage $4000 \mathrm{~V}$; gas temperature, $350{ }^{\circ} \mathrm{C}$; during gas $\mathrm{N}_{2}$ flow rate, $10 \mathrm{~L} \mathrm{~min}{ }^{-1}$; pressure of nebulizer, 35 psi. The mass analyzer scanned over m/z 100-3000 Da.

\subsection{Purification of transformation products (1-6)}

$5 \mathrm{~g}$ of the MEL extract was subjected to silica gel column chromatography eluted stepwise with $\mathrm{MeOH} / \mathrm{CHCl}_{3}$ (2:98, $5: 95,7: 93,10: 90,15: 85,20: 80,25: 75,30: 70,40: 60, \mathrm{v} / \mathrm{v})$ to afford 5 fractions (Fr. 1-5). Fr. 1 and Fr. 2 were purified by recrystallization to yield compound $\mathbf{1}(126.2 \mathrm{mg})$ and compound 2 (60 mg), respectively. Fr. 3 was subjected to pre-HPLC [YMC-


(B) $/ \mathrm{H}_{2} \mathrm{O}$ (A) (0-20 $\mathrm{min}, 40-50 \% \mathrm{~B} ; 20-65 \mathrm{~min}, 50-50 \% \mathrm{~B}$ ) mobile phase] to give compound $3\left(12.8 \mathrm{mg}, t_{\mathrm{R}}=54.0 \mathrm{~min}\right)$. Fr. 4 was successively purified by pre-HPLC [YMC-Pack ODS-A $(\phi 10 \times 250$ $\mathrm{mm}$ ), $2 \mathrm{~mL} \min ^{-1}$ flow rate, $\mathrm{MeCN}(\mathrm{B}) / \mathrm{H}_{2} \mathrm{O}$ (A) (0-40 min, 50$55 \% \mathrm{~B} ; 40-60 \mathrm{~min}, 55-55 \% \mathrm{~B})$ mobile phase] to yield compounds 4 (9.8 mg, $\left.t_{\mathrm{R}}=54.2 \mathrm{~min}\right)$ and $5\left(40.5 \mathrm{mg}, t_{\mathrm{R}}=58.4\right.$ $\min )$. Compounds $6\left(11.6 \mathrm{mg}, t_{\mathrm{R}}=58.5 \mathrm{~min}\right)$ was obtained from Fr. 5 separated by pre-HPLC [YMC-Pack ODS-A $(\phi 10 \times 250 \mathrm{~mm})$, $2 \mathrm{~mL} \min ^{-1}$ flow rate, $\mathrm{MeCN} / \mathrm{H}_{2} \mathrm{O}(55: 45)$ mobile phase].

2.4.1 20(S)-ginsenoside $\mathbf{R g}_{3}(\mathbf{1})$. White amorphous powder; HRESIMS $m / z 807.4663[\mathrm{M}+\mathrm{Na}]^{+} .{ }^{1} \mathrm{H}-\mathrm{NMR}\left(\mathrm{C}_{5} \mathrm{D}_{5} \mathrm{~N}, 500 \mathrm{MHz}\right) \delta$ : 0.94 (3H, s, H-18), 0.82 (3H, s, H-19), 1.40 (1H, s, H-21), 1.60 (3H, s, H-26), 1.60 (3H, s, H-27), 1.30 (3H, s, H-28), 1.11 (3H, s, H29), 0.99 ( $3 \mathrm{H}, \mathrm{s}, \mathrm{H}-30), 4.96$ (1H, d, $J=7.8 \mathrm{~Hz}$, Glc-I-H-1' $), 5.38$ $\left(1 \mathrm{H}, \mathrm{d}, J=7.7 \mathrm{~Hz}\right.$, Glc-II-H-1"). ${ }^{13} \mathrm{C}-\mathrm{NMR}\left(\mathrm{C}_{5} \mathrm{D}_{5} \mathrm{~N}, 125 \mathrm{MHz}\right) \delta$ : 39.5(C-1), 27.1(C-2), 89.3(C-3), 40.1(C-4), 56.7(C-5), 18.8(C-6), 35.5(C-7), 40.4(C-8), 50.8(C-9), 37.3(C-10), 32.4(C-11), 71.4(C12), 48.9(C-13), 52.1(C-14), 31.7(C-15), 27.2(C-16), 55.2(C-17), 16.2(C-18), 16.7(C-19), 73.3(C-20), 27.5(C-21), 36.3(C-22), 23.4(C-23), 126.7(C-24), 131.1(C-25), 26.2(C-26), $17.4(\mathrm{C}-27)$, 28.5(C-28), 17.0(C-29), 18.1(C-30), 105.5 (Glc-I-C-1'), 83.9 (C-2'), $78.4\left(\mathrm{C}-3^{\prime}\right), 72.1\left(\mathrm{C}-4^{\prime}\right), 78.7\left(\mathrm{C}-5^{\prime}\right), 63.2\left(\mathrm{C}-6^{\prime}\right), 106.5$ (Glc-II-C-1" $\left.{ }^{\prime \prime}\right)$, $77.5\left(\mathrm{C}-2^{\prime \prime}\right), 78.7\left(\mathrm{C}-3^{\prime \prime}\right), 72.0\left(\mathrm{C}-4^{\prime \prime}\right), 78.5\left(\mathrm{C}-5^{\prime \prime}\right), 63.1\left(\mathrm{C}-6^{\prime \prime}\right)$. The structure of compound 1 was identified as $20(S)$-ginsenoside $\mathrm{Rg}_{3}$ by comparing the NMR data and MS data with reported data. ${ }^{18}$

2.4.2 20(R)-ginsenoside $\mathbf{R g}_{3}$ (2). White amorphous powder; HRESIMS $m / z$ 807.4652 [M + Na $]^{+}$. ${ }^{1} \mathrm{H}-\mathrm{NMR}\left(\mathrm{C}_{5} \mathrm{D}_{5} \mathrm{~N}, 500 \mathrm{MHz}\right) \delta$ : 1.01 (3H, s, H-18), 0.82 (3H, s, H-19), 1.39 (1H, s, H-21), 1.69 (3H, s, H-26), 1.65 (3H, s, H-27), 1.30 (3H, s, H-28), 1.11 (3H, s, H29), 0.99 (3H, s, H-30), 4.94 (1H, d, $J=7.8 \mathrm{~Hz}$, Glc-I-H-1'), 5.38 $(1 \mathrm{H}, \mathrm{d}, J=7.7 \mathrm{~Hz}$, Glc-II-H-1" $) .{ }^{13} \mathrm{C}-\mathrm{NMR}\left(\mathrm{C}_{5} \mathrm{D}_{5} \mathrm{~N}, 125 \mathrm{MHz}\right) \delta$ : 39.1(C-1), 26.6(C-2), 88.9(C-3), 39.7(C-4), 56.4(C-5), 18.4(C-6), 35.1(C-7), 39.9(C-8), 50.3(C-9), 36.9(C-10), 31.3(C-11), 70.9(C12), 49.2(C-13), 51.8(C-14), 32.2(C-15), 26.7(C-16), 50.6(C-17), 15.8(C-18), 16.4(C-19), 73.0(C-20), 22.8(C-21), 43.2(C-22), 22.6(C-23), 126.0(C-24), 130.8(C-25), 25.9(C-26), 17.3(C-27), 28.2(C-28), 16.6(C-29), 17.7(C-30), 105.2 (Glc-I-C-1'), 83.5 (C-2'),
$78.0\left(\mathrm{C}-3^{\prime}\right), 71.6\left(\mathrm{C}-4^{\prime}\right), 78.3\left(\mathrm{C}-5^{\prime}\right), 62.9\left(\mathrm{C}^{\prime} 6^{\prime}\right), 106.1$ (Glc-II-C-1"), $77.2\left(\mathrm{C}-2^{\prime \prime}\right), 78.4\left(\mathrm{C}-3^{\prime \prime}\right), 71.6\left(\mathrm{C}-4^{\prime \prime}\right), 78.2\left(\mathrm{C}-5^{\prime \prime}\right), 62.7\left(\mathrm{C}-6^{\prime \prime}\right)$. The structure of compound 2 was identified as $20(R)$-ginsenoside $\mathrm{Rg}_{3}$ by comparing the NMR data and MS data with reported data. ${ }^{18}$

2.4.3 Notoginsenoside $\mathbf{S F t}_{3}$ (3). White amorphous powder; HRESIMS $m / z$ 921.4989 $[\mathrm{M}+\mathrm{Na}]^{+} .{ }^{1} \mathrm{H}-\mathrm{NMR}\left(\mathrm{C}_{5} \mathrm{D}_{5} \mathrm{~N}, 500 \mathrm{MHz}\right) \delta$ : 0.95 (3H, s, H-18), 0.79 (3H, s, H-19), 5.14 (1H, s, H-21), 4.90 (1H, s, H-21), 1.65 (3H, s, H-26), 1.59 (3H, s, H-27), 1.28 (3H, s, H28), 1.10 (3H, s, H-29), 1.00 (3H, s, H-30), 4.94 (1H, d, J = 7.8 Hz, Glc-I-H-1') $5.52\left(1 \mathrm{H}, \mathrm{d}, J=7.7 \mathrm{~Hz}\right.$, Glc-II-H-1 $\left.{ }^{\prime \prime}\right), 5.41(1 \mathrm{H}, \mathrm{d}, J=$ $6.7 \mathrm{~Hz}$, Xyl-H-1"' $) .{ }^{13} \mathrm{C}-\mathrm{NMR}\left(\mathrm{C}_{5} \mathrm{D}_{5} \mathrm{~N}, 125 \mathrm{MHz}\right) \delta: 39.8(\mathrm{C}-1)$, 26.8(C-2), 88.8(C-3), 39.3(C-4), 56.4(C-5), 18.5(C-6), 35.4(C-7), 40.3(C-8), 48.3(C-9), 37.1(C-10), 32.7(C-11), 72.4(C-12), 52.5(C13), 51.2(C-14), 32.8(C-15), 30.8(C-16), 50.8(C-17), 16.6(C-18), 15.9(C-19), 155.6(C-20), 108.2(C-21), 33.9(C-22), 27.1(C-23), 125.3(C-24), 131.2(C-25), 25.8(C-26), 17.8(C-27), 28.1(C-28), 16.7(C-29), 17.0(C-30), 104.8 (Glc-I-C-1' $), 83.0$ (C-2'), 78.7 (C-3'), $71.1\left(\mathrm{C}-4^{\prime}\right), 78.3\left(\mathrm{C}-5^{\prime}\right), 63.0\left(\mathrm{C}-6^{\prime}\right), 103.2$ (Glc-II-C-1" $), 84.5\left(\mathrm{C}-2^{\prime \prime}\right)$, $78.0\left(\mathrm{C}-3^{\prime \prime}\right), 71.8\left(\mathrm{C}-4^{\prime \prime}\right), 77.9\left(\mathrm{C}-5^{\prime \prime}\right), 62.8\left(\mathrm{C}-6^{\prime \prime}\right), 106.5$ (Xyl-C-1"' $)$, $76.0\left(\mathrm{C}-2^{\prime \prime \prime}\right), 77.8\left(\mathrm{C}-3^{\prime \prime \prime}\right), 70.8\left(\mathrm{C}-4^{\prime \prime \prime}\right), 67.5\left(\mathrm{C}-5^{\prime \prime \prime}\right)$. The structure of compound 3 was identified as notoginsenoside $\mathrm{SFt}_{3}$ by comparing the NMR data and MS data with reported data. ${ }^{19}$

2.4.4 Ginsenoside $\mathbf{R k}_{\mathbf{1}}$ (4). White amorphous powder; HRESIMS $m / z 789.4595[\mathrm{M}+\mathrm{Na}]^{+} .{ }^{1} \mathrm{H}-\mathrm{NMR}\left(\mathrm{C}_{5} \mathrm{D}_{5} \mathrm{~N}, 500 \mathrm{MHz}\right) \delta$ : 1.00 (3H, s, H-18), 0.80 (3H, s, H-19), 5.14 (1H, s, H-21), 4.90 (1H, s, H-21), 1.65 (3H, s, H-26), 1.59 (3H, s, H-27), 1.29 (3H, s, H28), 1.10 (3H, s, H-29), 0.95 (3H, s, H-30), 4.93 (1H, d, J=7.8 Hz, Glc-I-H-1'), 5.37 (1H, d, $J=7.7 \mathrm{~Hz}$, Glc-II-H-1" $) .{ }^{13} \mathrm{C}-\mathrm{NMR}$ $\left(\mathrm{C}_{5} \mathrm{D}_{5} \mathrm{~N}, 125 \mathrm{MHz}\right) \delta: 39.6(\mathrm{C}-1), 27.2(\mathrm{C}-2), 89.2(\mathrm{C}-3), 40.1(\mathrm{C}-4)$, 56.7(C-5), 18.8(C-6), 35.7(C-7), 40.5(C-8), 48.6(C-9), 37.3(C-10), 33.1(C-11), 72.8(C-12), 52.9(C-13), 51.5(C-14), 32.9(C-15), 31.2(C-16), 51.2(C-17), 16.8(C-18), 16.1(C-19), 155.9(C-20), 108.5(C-21), 34.2(C-22), 27.4(C-23), 125.8(C-24), 131.5(C-25), 26.1(C-26), 18.1(C-27), 28.4(C-28), 17.0(C-29), 17.3(C-30), 105.5 $\left(\right.$ Glc-I-C-1 $\left.{ }^{\prime}\right), 83.8\left(\mathrm{C}-2^{\prime}\right), 78.7\left(\mathrm{C}-3^{\prime}\right), 72.0\left(\mathrm{C}-4^{\prime}\right), 78.3\left(\mathrm{C}-5^{\prime}\right), 63.0(\mathrm{C}-$ $\left.6^{\prime}\right), 106.4$ (Glc-II-C-1 $\left.{ }^{\prime \prime}\right), 77.6\left(\mathrm{C}-2^{\prime \prime}\right), 78.7\left(\mathrm{C}-3^{\prime \prime}\right), 72.0\left(\mathrm{C}-4^{\prime \prime}\right), 78.5$ $\left(\mathrm{C}-5^{\prime \prime}\right), 63.2\left(\mathrm{C}-6^{\prime \prime}\right)$. The structure of compound 4 was identified as ginsenoside $\mathrm{Rk}_{1}$ by comparing the NMR data and MS data with reported data. ${ }^{20}$

2.4.5 Ginsenoside $\mathbf{R g}_{5}$ (5). White amorphous powder; HRESIMS $m / z 789.4569[\mathrm{M}+\mathrm{Na}]^{+} .{ }^{1} \mathrm{H}-\mathrm{NMR}\left(\mathrm{C}_{5} \mathrm{D}_{5} \mathrm{~N}, 500 \mathrm{MHz}\right) \delta$ : 1.00 (3H, s, H-18), 0.80 (3H, s, H-19), 1.83 (3H, s, H-21), $5.52(1 \mathrm{H}$, $\mathrm{t}, J=6.8 \mathrm{~Hz}, \mathrm{H}-22), 5.25(1 \mathrm{H}, \mathrm{t}, J=6.8 \mathrm{~Hz}, \mathrm{H}-24), 1.65(3 \mathrm{H}, \mathrm{s}, \mathrm{H}-$ 26), 1.59 (3H, s, H-27), 1.29 (3H, s, H-28), 1.10 (3H, s, H-29), 0.95 $(3 \mathrm{H}, \mathrm{s}, \mathrm{H}-30), 4.93\left(1 \mathrm{H}, \mathrm{d}, J=7.8 \mathrm{~Hz}\right.$, Glc-I-H-1 $\left.{ }^{\prime}\right), 5.37(1 \mathrm{H}, \mathrm{d}, J=$ $7.7 \mathrm{~Hz}$, Glc-II-H-1" $) .{ }^{13} \mathrm{C}-\mathrm{NMR}\left(\mathrm{C}_{5} \mathrm{D}_{5} \mathrm{~N}, 125 \mathrm{MHz}\right) \delta: 39.6(\mathrm{C}-1)$, 28.5(C-2), 89.3(C-3), 40.6(C-4), 56.7(C-5), 18.8(C-6), 35.7(C-7), 40.3(C-8), 51.1(C-9), 37.3(C-10), 33.1(C-11), 72.8(C-12), 50.9(C13), 51.5(C-14), 32.9(C-15), 31.1(C-16), 51.2(C-17), 16.8(C-18), 17.0(C-19), 140.5(C-20), 13.5(C-21), 123.5(C-22), 27.7(C-23), 123.8(C-24), 131.5(C-25), 26.1(C-26), 18.1(C-27), 28.9(C-28), 16.1(C-29), 17.3(C-30), 105.5 (Glc-I-C-1' $), 83.8$ (C-2'), 78.7 (C-3'), $72.0\left(\mathrm{C}-4^{\prime}\right), 78.3\left(\mathrm{C}-5^{\prime}\right), 63.0\left(\mathrm{C}-6^{\prime}\right), 106.4$ (Glc-II-C-1" $), 77.6\left(\mathrm{C}-2^{\prime \prime}\right)$, $78.7\left(\mathrm{C}-3^{\prime \prime}\right), 72.0\left(\mathrm{C}-4^{\prime \prime}\right), 78.5\left(\mathrm{C}-5^{\prime \prime}\right), 63.2\left(\mathrm{C}-6^{\prime \prime}\right)$. The structure of compound 5 was identified as ginsenoside $\mathrm{Rg}_{5}$ by comparing the NMR data and MS data with reported data. ${ }^{21}$ 
2.4.6 20(S)-ginsenoside $\mathbf{R h}_{2}(\mathbf{6})$. White amorphous powder; HRESIMS $m / z 1245.8682[2 \mathrm{M}+\mathrm{H}]^{+} .{ }^{1} \mathrm{H}-\mathrm{NMR}\left(\mathrm{C}_{5} \mathrm{D}_{5} \mathrm{~N}, 500 \mathrm{MHz}\right)$ $\delta: 0.95(3 \mathrm{H}, \mathrm{s}, \mathrm{H}-18), 0.78$ (3H, s, H-19), $1.42(1 \mathrm{H}, \mathrm{s}, \mathrm{H}-21), 1.61$ (3H, s, H-26), 1.64 (3H, s, H-27), 1.31 (3H, s, H-28), 0.99 (3H, s, H29), 0.95 (3H, s, H-30), 4.96 (1H, d, $J=7.8 \mathrm{~Hz}$, Glc-I-H-1 $) .{ }^{13} \mathrm{C}-$ NMR $\left(\mathrm{C}_{5} \mathrm{D}_{5} \mathrm{~N}, 125 \mathrm{MHz}\right) \delta$ : 39.1(C-1), 26.9(C-2), 88.8(C-3), 39.7(C-4), 56.4(C-5), 18.5(C-6), 35.2(C-7), 40.0(C-8), 50.3(C-9), 37.0(C-10), 32.1(C-11), $\quad 71.0(\mathrm{C}-12), \quad 48.5(\mathrm{C}-13), \quad 51.8(\mathrm{C}-14)$, 31.3(C-15), 26.7(C-16), 54.8(C-17), 16.4(C-18), 15.8(C-19), 73.0(C-20), 27.1(C-21), 35.9(C-22), 23.0(C-23), 126.3(C-24), 130.8(C-25), 25.8(C-26), 17.7(C-27), 28.2(C-28), 16.8(C-29), 17.1(C-30), 107.0 (Glc-C-1'), 75.8 (C-2'), 78.8 (C-3'), 71.9 (C-4'), $78.4\left(\mathrm{C}-5^{\prime}\right), 63.1\left(\mathrm{C}-6^{\prime}\right)$. The structure of compound 6 was identified as $20(S)$-ginsenoside $\mathrm{Rh}_{2}$ by comparing the NMR data and MS data with reported data. ${ }^{21}$

\subsection{HPLC analysis of transformation product (1-6)}

Quantitative analysis of six transformation product (1-6) processed by microwave processing method was determined by high-performance liquid chromatography (HPLC) (Fig. 3). The HPLC data were recorded on a Shimadzu Analytical Instrument (Shimadzu, Kyoto, Japan), equipped with a solvent degasser, a binary pump, an auto sampler and a UV detector. The separation was performed on a VisionHT C18 HL column $(250 \mathrm{~mm}$ $\times 4.6 \mathrm{~mm}, 5 \mu \mathrm{m}$, Grace, Deerfield, USA) at the flow rate of 1.0 $\mathrm{mL} \min ^{-1}$. The mobile phase consisted of distilled water (solvent A) and acetonitrile (solvent B) using the following gradient program: $0-20 \mathrm{~min}, 20-20 \% \mathrm{~B} ; 20-45 \mathrm{~min}, 20-46 \% \mathrm{~B}$; 45-50 min, 46-55\% B; 50-66 min, 55-55\% B. The detection wavelength and the column temperature were at $203 \mathrm{~nm}$ and $35{ }^{\circ} \mathrm{C}$, respectively. The calibration curves for $20(S)-\mathrm{Rg}_{3}(\mathbf{1})$, $20(R)-\mathrm{Rg}_{3}(2), \mathrm{SFt}_{3}(3), \mathrm{Rk}_{1}(4), \mathrm{Rg}_{5}(5)$ and $20(S)-\mathrm{Rh}_{2}(\mathbf{6})$ showed good linearity $\left(R^{2}>0.9990\right)$ in the concentration ranges (Table 1). The samples were analyzed by HPLC in three times.

\subsection{Anticancer activity in vitro}

2.6.1 Cell lines and culture. Human cervical cancer (HeLa) and human lung cancer (A549) cell lines were obtained from the Cell Bank of Shanghai Institutes for Biological Sciences, Chinese Academy of Sciences. HeLa cells and A549 cells were cultivated in RPMI Media 1640 (RPMI1640) containing 10\% fetal bovine serum, $100 \mathrm{U} \mathrm{mL}^{-1}$ penicillin, $0.1 \mathrm{mg} \mathrm{mL}^{-1}$ streptomycin and $2 \mathrm{mmol} \mathrm{L}^{-1} \mathrm{~L}$-glutamine. All cultures were maintained with the aeration of $5 \% \mathrm{CO}_{2}$ at $37^{\circ} \mathrm{C}$.

2.6.2 Cell cytotoxicity assay. The cytotoxicity of EL, MEL and compounds 1-6 on two cancer cell lines was determined by MTT assay. ${ }^{22}$ The cells were seeded in five-well plates $\left(1 \times 10^{4}\right.$ cells per well) and incubated at $37^{\circ} \mathrm{C}$ for $24 \mathrm{~h}$. The cells were then treated with EL, MEL, cisplatin and compounds 1-6 at different concentrations $\left(0.1-100 \mu \mathrm{mol} \mathrm{L}^{-1}\right)$. Cisplatin was used as the positive control. After $48 \mathrm{~h}, 20 \mu \mathrm{L}$ MTT $\left(5 \mathrm{mg} \mathrm{mL}^{-1}\right)$ was added to each well and the cells were incubated for $4 \mathrm{~h}$. The supernatant was removed and then $150 \mu \mathrm{L}$ of DMSO was added to each well. After the plates were shaken at room temperature for $10 \mathrm{~min}$, the absorbance was measured by PHERA star FSX Microplate Reader (BMG Labtech, Offenburg, Germany) at a wavelength of $570 \mathrm{~nm}$. The half maximal inhibitory concentration $\left(\mathrm{IC}_{50}\right)$ against cancer cells was obtained by curve fitting of a sigmoidal dosage-response curve using nonlinear regression model.

\subsection{Anticoagulant activity in vitro}

2.7.1 Animals. Kunming mice (18-22 g) were purchased by Changsha Tianqin Biotechnology Co., China (Qualified no. SCXK 2014-0011). The mice were maintained at a temperature of $25 \pm 2{ }^{\circ} \mathrm{C}$, a humidity of $62 \pm 2 \%$, and a $12 \mathrm{~h}$ dark/light cycle, with unrestricted access to food and water. All animal procedures were performed in accordance with the Guidelines for Care and Use of Laboratory Animals of Kunming University of Science and Technology, and were approved by the Animal Ethics Committee of Kunming University of Science and Technology.

2.7.2 Plasma preparation. The plasma was obtained from mice whole blood mixed with $0.109 \mathrm{~mol} \mathrm{~L}^{-1}$ sodium citrate $(9: 1$ ratio of blood to citrate, $\mathrm{v} / \mathrm{v})$ by centrifugation at $3000 \times g$ for $15 \mathrm{~min}$.

2.7.3 Blood plasma clotting assays. Prothrombin time (PT) was determined with a coagulation analyzer (XN06-IV, Aierfu, Wuhan, China). $100 \mu \mathrm{L}$ of a solution containing plasma $(50 \mu \mathrm{L})$ and EL, MEL and compounds 1-6 $(50 \mu \mathrm{L})$ were incubated at $37^{\circ} \mathrm{C}$ for $3 \mathrm{~min}$. The samples of EL and MEL were diluted with purified water to give the following concentrations: $25,12.5$, $5 \mathrm{mg} \mathrm{mL}{ }^{-1}$. The concentration of compounds 1-6 is $1 \mathrm{mg} \mathrm{mL}^{-1}$. Purified water was used as negative control. The PT assay reagent $(100 \mu \mathrm{L})$ was added to the mixed samples, and the clotting time was recorded by the analyzer. All clotting tests were performed with three individual replicates.

\subsection{Statistical analysis}

Statistical analyses were performed with SPSS 19.0 software. All data were expressed as mean \pm standard deviation (SD). The values of $p<0.05$ were considered to be statistically significant, and $p<0.01$ and $p<0.001$ being very significant.

\section{Results and discussion}

3.1 Purification and identification of the transformation products from the stems and leaves of $P$. notoginseng using the microwave processing method

The MEL was identified by ultra-high performance liquid chromatography-quantitative time of flight mass spectroscopy (QTOF-MS) in positive mode. The total ion chromatogram of the MEL is shown in Fig. 4. Table 2 summarizes the QTOF-MS data of 22 ginsenosides identified from the MEL. The compounds were determined by the molecular ions based on $[\mathrm{M}+\mathrm{H}]^{+},[\mathrm{M}+\mathrm{Na}]^{+}$, or $[2 \mathrm{M}+\mathrm{H}]^{+}$. The chemical composition of the MEL was mainly 20(S)-protopanaxadiol (PPD)-type ginsenosides; the PPD skeleton of which displayed characteristic ions at $m / z 425 .{ }^{23,24}$ Eight transformation products in the MEL were identified as ginsenosides, such as $\mathrm{F}_{2}, 20(S)-\mathrm{Rg}_{3}, 20(R)$ $\mathrm{Rg}_{3}, \mathrm{SFt}_{3}, \mathrm{Rk}_{1}, \mathrm{Rg}_{5}, 20(S)-\mathrm{Rh}_{2}$, and $20(R)-\mathrm{Rh}_{2}$. The others had already existed in the PNL before microwave transformation, 
A



C



E

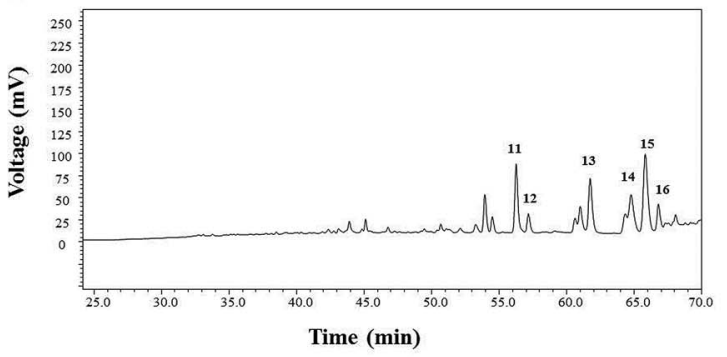

G

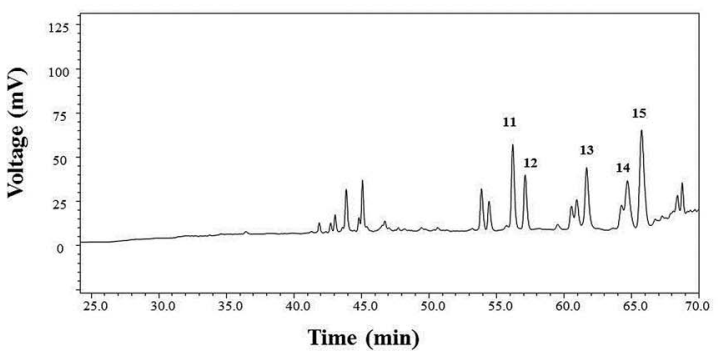

B

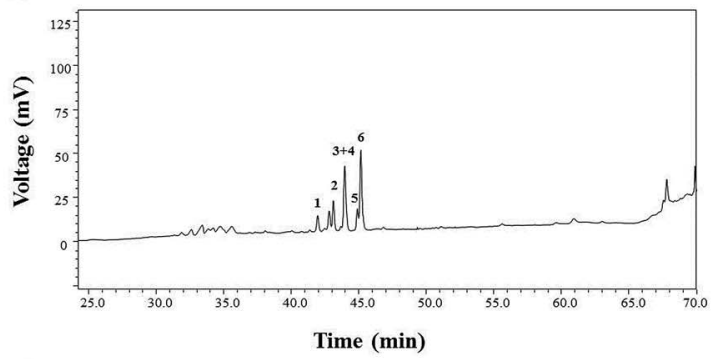

D

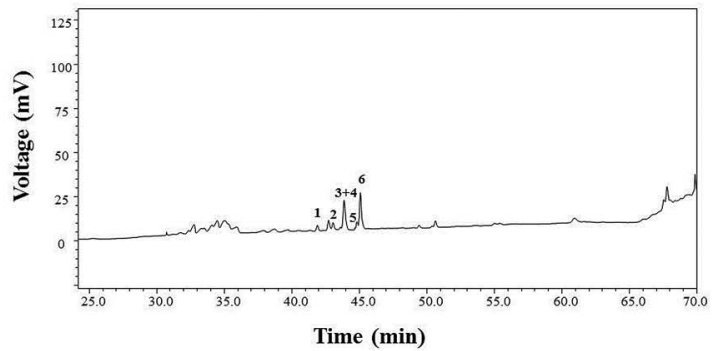

F



H

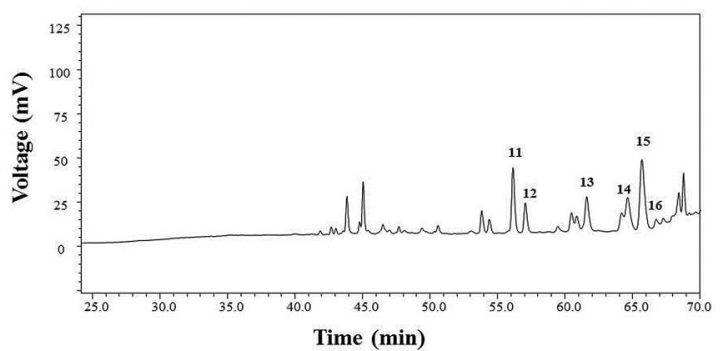

Fig. 3 Saponin transformation in different parts of Panax notoginseng induced by microwave processing. HPLC chromatograms of the stems and leaves (A), flowers (B), pedicels (C), and seeds (D) of P. notoginseng. HPLC chromatograms of the stems and leaves (E), flowers (F), pedicels $(G)$, and seeds $(H)$ of $P$. notoginseng after treatment with microwave processing. Peaks: (1) notoginsenoside Fa; (2) ginsenoside Rb1; (3) ginsenoside Rc; (4) notoginsenoside Fc; (5) ginsenoside Rb2; (6) ginsenoside Rb3; (7) ginsenoside Rd; (8) notoginsenoside Fe; (9) ginsenoside Rd2; (10) gypenoside IX; (11) 20(S)-ginsenoside Rg3; (12) 20(R)-ginsenoside Rg3; (13) notoginsenoside SFt3; (14) ginsenoside Rk1; (15) ginsenoside Rg5; (16) $20(S)$-ginsenoside Rh2.

Table 1 The results of linear regression of transformation product $(1-6)(n=6)$

Compounds

Ginsenoside $20(S)-\operatorname{Rg}_{3}(\mathbf{1})$

Ginsenoside $20(R)-\mathrm{Rg}_{3}(2)$

Notoginsenoside $\mathrm{SFt}_{3}(3)$

Ginsenoside $\mathrm{Rk}_{1}(\mathbf{4})$

Ginsenoside $\operatorname{Rg}_{5}(5)$

Ginsenoside $20(S)-\mathrm{Rh}_{2}(6)$

\section{Calibration curve}

$y=4000000 x+9678$

$y=4000000 x+15317$

$y=990240 x+37027$

$y=3000000 x+1465$

$y=758099 x+45996$

$y=3000000 x+10426$
Concentration range $\left(\mu \mathrm{g} \mathrm{mL}{ }^{-1}\right)$

$1.0-1000$

4.0-1000

3.1-3000

$4.0-1000$

16.0-5000

$2.5-1000$ 


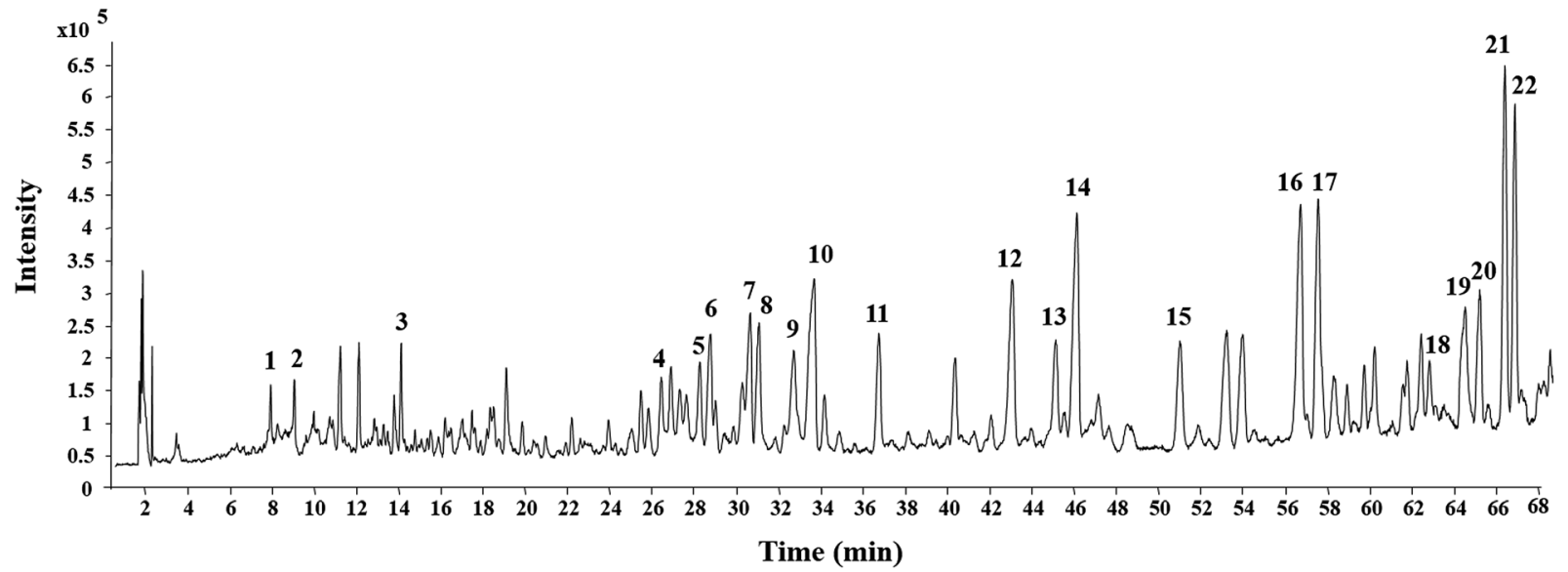

Fig. 4 Total ion current chromatogram of microwave processing extract of the stems and leaves of Panax notoginseng by UHPLC-ESI-Q-TOF-MS.

Table 2 Characterization of ginsenosides in microwave processing extract of stems and leaves of $P$. notoginseng using UHPLC-Q-TOF/MS

\begin{tabular}{|c|c|c|c|c|c|}
\hline No. & $\begin{array}{l}\text { Retention time } \\
(\mathrm{min})\end{array}$ & Experimental $\mathrm{m} / \mathrm{z}$ & Molecular formula & Diagnostic fragments $(\mathrm{m} / \mathrm{z})$ & Identification \\
\hline 1 & 7.31 & $627.1402[\mathrm{M}+\mathrm{H}]^{+}$ & $\mathrm{C}_{42} \mathrm{H}_{26} \mathrm{O}_{6}$ & $\begin{array}{l}\text { 303.0410, 333.0460, 465.0912, 515.2338, } \\
531.2084,566.4141,588.3944\end{array}$ & Unknown \\
\hline 2 & 8.47 & $611.1461[\mathrm{M}+\mathrm{H}]^{+}$ & $\mathrm{C}_{42} \mathrm{H}_{26} \mathrm{O}_{5}$ & $287.0467,449.0962,566.4133,588.3948$ & Unknown \\
\hline 3 & 13.54 & $823.4602[\mathrm{M}+\mathrm{Na}]^{+}$ & $\mathrm{C}_{42} \mathrm{H}_{72} \mathrm{O}_{14}$ & $\begin{array}{l}423.3510,441.3612,603.4086,621.4228 \\
765.4602\end{array}$ & Ginsenoside $\mathrm{Rg}_{1}$ \\
\hline 4 & 25.92 & $1263.6069[\mathrm{M}+\mathrm{Na}]^{+}$ & $\mathrm{C}_{59} \mathrm{H}_{100} \mathrm{O}_{27}$ & $\begin{array}{l}425.3665,587.4160,605.4256,749.4662 \\
881.5030\end{array}$ & Notoginsenoside Fa \\
\hline 5 & 27.67 & $1233.5957[\mathrm{M}+\mathrm{Na}]^{+}$ & $\mathrm{C}_{58} \mathrm{H}_{98} \mathrm{O}_{26}$ & $\begin{array}{l}\text { 425.3659, 603.4095, 749.4646, 823.4584, } \\
881.5069,955.5007,1083.5334\end{array}$ & Ginsenoside $\mathrm{FP}_{2}$ \\
\hline 6 & 28.21 & $1131.5648[\mathrm{M}+\mathrm{Na}]^{+}$ & $\mathrm{C}_{54} \mathrm{H}_{92} \mathrm{O}_{23}$ & $425.3658,587.4171,749.4646,929.5196$ & Ginsenoside $\mathrm{Rb}_{1}$ \\
\hline 7 & 30.08 & $1101.554[\mathrm{M}+\mathrm{Na}]^{+}$ & $\mathrm{C}_{53} \mathrm{H}_{90} \mathrm{O}_{22}$ & $\begin{array}{l}425.3664,587.4152,605.4269,749.4670 \\
899.5157\end{array}$ & Ginsenoside $\mathrm{R}_{\mathrm{c}}$ \\
\hline 8 & 30.48 & $1233.5946[\mathrm{M}+\mathrm{Na}]^{+}$ & $\mathrm{C}_{58} \mathrm{H}_{98} \mathrm{O}_{26}$ & $\begin{array}{l}\text { 425.3666, 587.4158, 605.4267, 749.4656, } \\
881.5080,916.5333\end{array}$ & Notoginsenoside Fc \\
\hline 9 & 32.15 & $1101.5561[\mathrm{M}+\mathrm{Na}]^{+}$ & $\mathrm{C}_{53} \mathrm{H}_{90} \mathrm{O}_{22}$ & $\begin{array}{l}\text { 425.3664, 559.2657, 605.4261, 749.4647, } \\
783.4688,881.5043\end{array}$ & Ginsenoside $\mathrm{Rb}_{2}$ \\
\hline 10 & 33.01 & $1101.5591[\mathrm{M}+\mathrm{Na}]^{+}$ & $\mathrm{C}_{53} \mathrm{H}_{90} \mathrm{O}_{22}$ & $\begin{array}{l}\text { 425.3666, 587.4169, 605.4273, 749.4655, } \\
881.5060,929.5193\end{array}$ & Ginsenoside $\mathrm{Rb}_{3}$ \\
\hline 11 & 36.19 & $969.5170[\mathrm{M}+\mathrm{Na}]^{+}$ & $\mathrm{C}_{48} \mathrm{H}_{82} \mathrm{O}_{18}$ & $425.3663,587.4159,767.4730$ & Ginsenoside $R_{d}$ \\
\hline 12 & 42.46 & $939.5082[\mathrm{M}+\mathrm{Na}]^{+}$ & $\mathrm{C}_{47} \mathrm{H}_{80} \mathrm{O}_{17}$ & $\begin{array}{l}425.3672,587.4170,605.4255,719.4548 \\
737.4652,749.4659,767.4736,793.4525 \\
881.5041\end{array}$ & Notoginsenoside Fe \\
\hline 13 & 44.55 & $939.5077[\mathrm{M}+\mathrm{Na}]^{+}$ & $\mathrm{C}_{47} \mathrm{H}_{80} \mathrm{O}_{17}$ & $\begin{array}{l}425.3673,587.4156,605.4274,719.4553 \\
737.4644,749.4686,767.4758,785.4860 \\
881.5065\end{array}$ & Ginsenoside $\mathrm{Rd}_{2}$ \\
\hline 14 & 45.54 & $939.5067[\mathrm{M}+\mathrm{Na}]^{+}$ & $\mathrm{C}_{47} \mathrm{H}_{80} \mathrm{O}_{17}$ & $\begin{array}{l}425.3670,587.4161,605.4256,719.4560 \\
737.4651,749.4665,767.4736,881.5061\end{array}$ & Notoginsenoside Fd \\
\hline 15 & 50.42 & $807.4665[\mathrm{M}+\mathrm{Na}]^{+}$ & $\mathrm{C}_{42} \mathrm{H}_{72} \mathrm{O}_{13}$ & $\begin{array}{l}425.3670,587.4160,605.4252,749.4644 \\
765.4599\end{array}$ & Ginsenoside $\mathrm{F}_{2}$ \\
\hline 16 & 56.17 & $807.4663[\mathrm{M}+\mathrm{Na}]^{+}$ & $\mathrm{C}_{42} \mathrm{H}_{72} \mathrm{O}_{13}$ & $425.3663,587.4155,605.4249,749.4658$ & 20(S)-Ginsenoside $\mathrm{Rg}_{3}$ \\
\hline 17 & 57.01 & $807.4652[\mathrm{M}+\mathrm{Na}]^{+}$ & $\mathrm{C}_{42} \mathrm{H}_{72} \mathrm{O}_{13}$ & $425.3667,587.4162,605.4270,749.4646$ & $20(R)$-Ginsenoside $\mathrm{Rg}_{3}$ \\
\hline 18 & 61.89 & $921.4989[\mathrm{M}+\mathrm{Na}]^{+}$ & $\mathrm{C}_{47} \mathrm{H}_{78} \mathrm{O}_{16}$ & $\begin{array}{l}\text { 407.3562, 425.3663, 587.4162, 605.4257, } \\
749.4642,881.5078\end{array}$ & Ginsenoside $\mathrm{SFt}_{3}$ \\
\hline 19 & 63.94 & $789.4595[\mathrm{M}+\mathrm{Na}]^{+}$ & $\mathrm{C}_{42} \mathrm{H}_{70} \mathrm{O}_{12}$ & $\begin{array}{l}407.3559,425.3664,587.4159,605.4272 \\
749.4663\end{array}$ & Ginsenoside $\mathrm{Rk}_{1}$ \\
\hline 20 & 64.67 & $789.4569[\mathrm{M}+\mathrm{Na}]^{+}$ & $\mathrm{C}_{42} \mathrm{H}_{70} \mathrm{O}_{12}$ & $\begin{array}{l}407.3563,425.3663,587.4163,605.4263 \\
749.4657\end{array}$ & Ginsenoside $\mathrm{Rg}_{5}$ \\
\hline 21 & 65.85 & $1245.8682[2 \mathrm{M}+\mathrm{H}]^{+}$ & $\mathrm{C}_{36} \mathrm{H}_{62} \mathrm{O}_{8}$ & $407.3562,425.3663,587.4156,605.4254$ & 20(S)-Ginsenoside $\mathrm{Rh}_{2}$ \\
\hline 22 & 66.34 & $1245.8688[2 \mathrm{M}+\mathrm{H}]^{+}$ & $\mathrm{C}_{36} \mathrm{H}_{62} \mathrm{O}_{8}$ & $407.3559,425.3669,587.4160,605.4269$ & $20(R)$-Ginsenoside $\mathrm{Rh}_{2}$ \\
\hline
\end{tabular}


which were identified by matching molecular formulas with reported data., ${ }^{3,4}$ Six transformation products were purified from the MEL, and their structures were determined by comparing their spectroscopic data with those reported in the literature (Fig. 2). The transformation products were identified as 20(S)-ginsenoside $\mathrm{Rg}_{3}(\mathbf{1}){ }^{18} 20(R)$-ginsenoside $\operatorname{Rg}_{3}(2){ }^{18}$ notoginsenoside $\mathrm{SFt}_{3}(3),{ }^{19}$ ginsenoside $\mathrm{Rk}_{1}(4),{ }^{20}$ ginsenoside $\mathrm{Rg}_{5}(5),{ }^{20}$ and $20(S)$-ginsenoside $\mathrm{Rh}_{2}(6) .{ }^{21}$

\subsection{Impact of microwave processing conditions on the yields of the transformation products}

Microwave processing as a "green and innovative" technique has been widely used for preservation, ${ }^{25}$ transformation, ${ }^{\mathbf{1 3}, 14}$ and extraction $^{26}$ in research and industrial fields. The microwave processing technique has advantages of less time and energy as well as fewer solvents. ${ }^{16}$ In our study, this microwave processing method was applied to degrade the major saponins in the PNL and to produce the rare saponins with fewer sugar moieties. Microwave heating occurs within the molecule, and the glycosidic bonds in the polar molecules break easily. ${ }^{15}$ The major saponins in PNL were hydrolyzed at the glucosyl residue of C-20 to produce ginsenosides $20(S)-\mathrm{Rg}_{3}, 20(R)-\mathrm{Rg}_{3}, 20(S)-\mathrm{Rh}_{2}, \mathrm{Rk}_{1}$, $\mathrm{Rg}_{5}$, and notoginsenoside $\mathrm{SFt}_{3}$ in the MEL with an increase in microwave power and temperature. The three factors of microwave power, temperature, and time were assessed for yields of the five transformation products by HPLC analysis after microwave processing (Fig. 5).
Microwave power was an important factor in the transformation process. When microwave temperature and time were set to $135{ }^{\circ} \mathrm{C}$ and $15 \mathrm{~min}$, microwave power was varied from 300 to $1000 \mathrm{~W}$ to evaluate its impact on the yields of the transformation products (Fig. 5A). The highest yield of each transformation product was reached with microwave power of $500 \mathrm{~W}$. The yields of the five transformation products in the MEL were in the order of $\mathrm{Rg}_{5}>\mathrm{SFt}_{3}>20(R)-\mathrm{Rg}_{3}>20(S)-\mathrm{Rg}_{3}>$ $20(S)-\mathrm{Rh}_{2}$. When the microwave temperature range was from $60-180^{\circ} \mathrm{C}$ for $20 \mathrm{~min}$, the yields of the transformation products increased gradually (Fig. 5B). When the temperature was $150^{\circ} \mathrm{C}$, the yields of the transformation products were the highest in the following order: $\mathrm{Rg}_{5}>\mathrm{SFt}_{3}>20(R)-\mathrm{Rg}_{3}>20(S)-\mathrm{Rg}_{3}>20(S)$ $\mathrm{Rh}_{2}$. According to the yields of the transformation products, a suitable time for this microwave processing method was 15$30 \mathrm{~min}$ (Fig. 5C). The yields of the transformation products were in the following order: $\mathrm{Rg}_{5}>\mathrm{SFt}_{3}>20(R)-\mathrm{Rg}_{3}>20(S)-\mathrm{Rg}_{3}>20(S)$ $\mathrm{Rh}_{2}$. It was reported that the contents of the primary degradation products $20(S)-\mathrm{Rh}_{1}, 20(R)-\mathrm{Rh}_{1}, \mathrm{Rk}_{3}, \mathrm{Rh}_{4}, 20(S)-\mathrm{Rg}_{3}, 20(R)$ $\mathrm{Rg}_{3}, \mathrm{Rk}_{1}$, and $\mathrm{Rg}_{5}$ for dried notoginseng steamed at $120{ }^{\circ} \mathrm{C}$ increase rapidly after 12 h. ${ }^{12}$ A similar yield was achieved in 20 min when the microwave processing method was used. Transformation products are obtained by microwave processing with less energy and less waste compared with the steaming method. According to the yields of the transformation products after microwave processing, the optimum conditions of microwave power, temperature, and time were $500 \mathrm{~W}, 150{ }^{\circ} \mathrm{C}$, and $20 \mathrm{~min}$, respectively.
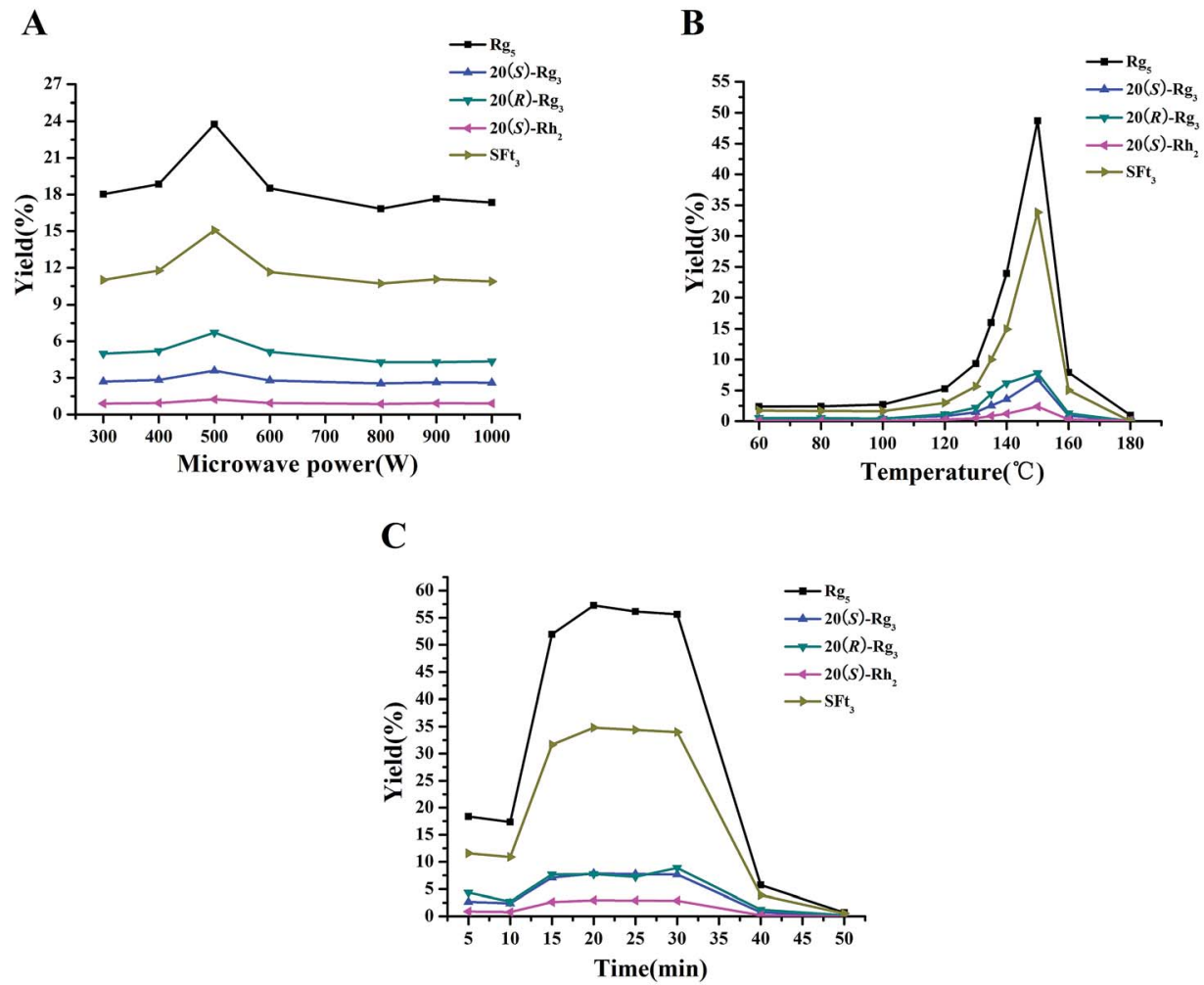

Fig. 5 Effects of microwave power (A), temperature (B), and time (C) on yields of transformation products. 


\subsection{Applying the microwave processing method to different parts of $P$. notoginseng}

The results of saponin transformation in different parts of $P$. notoginseng (stems and leaves, flowers, seeds, and pedicels) using microwave processing are shown in Fig. 3 and 6. High performance liquid chromatography chromatograms of stems and leaves, flowers, seeds, and pedicels of $P$. notoginseng revealed similar typical ginsenosides as the $\mathrm{PNL}$, consisting of notoginsenosides $\mathrm{Fa}$ and $\mathrm{Fc}$, and ginsenosides $\mathrm{Rc}, \mathrm{Rb}_{1}, \mathrm{Rb}_{2}$, and $\mathrm{Rb}_{3}$ (Fig. 3A-D). After microwave processing, six newly transformed products of $20(S)-\mathrm{Rg}_{3}, 20(R)-\mathrm{Rg}_{3}, \mathrm{SFt}_{3}, \mathrm{Rk}_{1}, \mathrm{Rg}_{5}$, and $20(S)-\mathrm{Rh}_{2}$ were generated from the MEL, flowers (MEF), seeds (MES), and pedicels (MEP) of P. notoginseng (Fig. 3E-H). As shown in Fig. 6, the $\mathrm{Rg}_{5}$ content in the microwave-treated extracts from different parts of $P$. notoginseng was the highest and the $\mathrm{Rh}_{2}$ content was the lowest. Under the same microwave processing conditions $\left(500 \mathrm{~W}, 500{ }^{\circ} \mathrm{C}\right.$, and $\left.20 \mathrm{~min}\right)$ the yields of $\mathrm{Rg}_{5}$ in the microwave transformed extracts from different parts of $P$. notoginseng were in the order of: MEL $>$ MEP $>$ MES $>$ MEF. The $20(S)-\mathrm{Rh}_{2}$ contents in the MEL and MES were $2.58 \%$ and $0.36 \%$ respectively, while $20(S)-\mathrm{Rh}_{2}$ was not detected in the MEP or MEF. Three special saponins of notoginsenoside Fe, ginsenoside $\mathrm{Rd}_{2}$, and gypenoside IX were detected in the PNL (Fig. 3A), which easily lost the glycosyl group at C-20 to generate $20(S)-\mathrm{Rh}_{2}$. This result indicates that $20(S)-\mathrm{Rh}_{2}$ content in the MEL was much higher than that in the MEP, MES, or MEF.

\subsection{Transformation mechanism for producing the minor ginsenosides using the microwave processing method}

Microwave heating occurs within molecules, which is described as "internal heating". ${ }^{15}$ If the microwave energy matches with the molecular bond energy, the reactive activity of glycoside bond in the molecule increased and easy to break..$^{15,27}$ In our paper, major saponins lost the glycosyl residues and produced the minor ginsenosides with increased microwave power and temperature. The proposed transformation pathway of

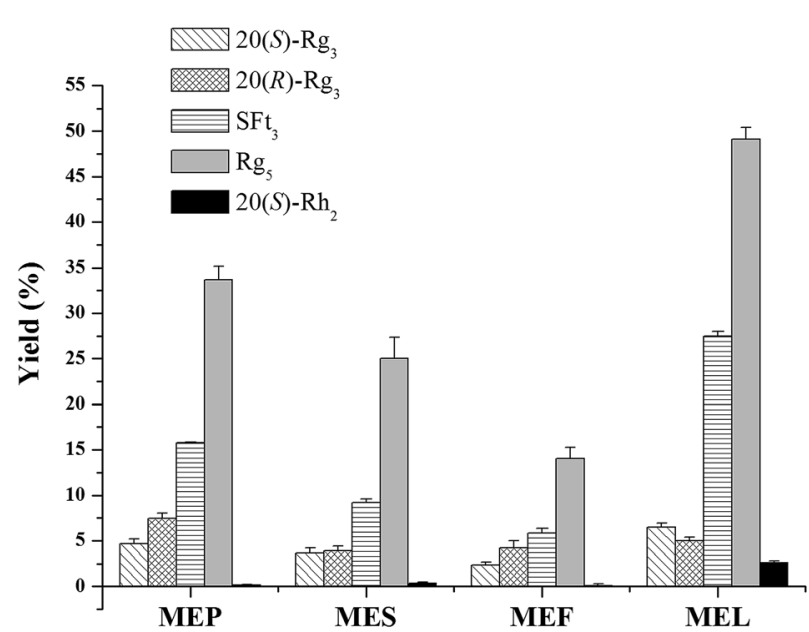

Fig. 6 The contents of five transformation products from microwave processing extracts of stems and leaves (MEL), flowers (MEF), seeds (MES), and pedicels (MEP) of $P$. notoginseng. Data are expressed as mean $\pm \mathrm{SD}(n=3)$. saponins in PNL by the microwave processing were shown in Fig. 7. The major saponins, e.g., ginsenosides $\mathrm{Rb}_{1}, \mathrm{Rd}, \mathrm{Rc}, \mathrm{Rb}_{2}$, and $\mathrm{Rb}_{3}$ easily lost the glycosyl residue at C-20 to produce $20(S) /$ $(R)-\mathrm{Rg}_{3}$, and then formed $\mathrm{Rk}_{1}$ and $\mathrm{Rg}_{5}$ through dehydration at C20. Notoginsenosides Fa and Fc were hydrolyzed and dehydrated at $\mathrm{C}-20$ to obtain notoginsenoside $\mathrm{SFt}_{3}$. When the microwave temperature was increased, a small amount of $20(S) /$ $(R)-\mathrm{Rg}_{3}$ was converted to $20(S) /(R)-\mathrm{Rh}_{2}$ through loss of a glucosyl group at C-3. In addition, the three compounds of notoginsenoside $\mathrm{Fe}$, ginsenoside $\mathrm{Rd}_{2}$ and gypenoside IX firstly removed the arabinose or xylose residues at C-3 to produce ginsenoside $\mathrm{F}_{2}$, and continued to lose one glucose residue at C-20 to obtain $20(S)-\mathrm{Rh}_{2}$. This indicated that the content of $20(S)-\mathrm{Rh}_{2}$ was much higher than that of $20(R)-\mathrm{Rh}_{2}$ in the microwave processing. When the glycosyl residues at C-20 were lost, $20(S / R)$ epimers such as $20(S / R)-\mathrm{Rg}_{3}$ and $20(S / R)-\mathrm{Rh}_{2}$ were obtained by the selective attack of the OH group. ${ }^{28}$

The structures of both $20(S / R)-\mathrm{Rg}_{3}$ and $20(S)-\mathrm{Rh}_{2}$ had been characterized with NMR. The ${ }^{13} \mathrm{C}$ NMR analysis suggested the obvious changes of the chemical shifts in $20(S / R)$ epimers at C$17, \mathrm{C}-21$ and C-22. The chemical shifts of $20(S)-\mathrm{Rg}_{3}$ were $\delta_{\mathrm{C}}$ 55.2(C-17), 27.5(C-21) and 36.3(C-22) ppm, while those of 20(R)$\mathrm{Rg}_{3}$ were $\delta_{\mathrm{C}} 50.6(\mathrm{C}-17), 22.8(\mathrm{C}-21)$ and $43.2(\mathrm{C}-22) \mathrm{ppm}$. The values in $20(S)-\mathrm{Rh}_{2}$ were also observed in the peaks of $\delta_{\mathrm{C}} 54.8(\mathrm{C}$ 17), 27.1(C-21) and 35.9(C-22) ppm. The results were in agreement with the reported data. ${ }^{18}$ After dehydration at $\Delta 20(21)$ or $\Delta 20(22)$, the values were shifted to $\delta_{\mathrm{C}} 155.75 \pm 0.15(\mathrm{C}-20)$ and $108.35 \pm 0.15(\mathrm{C}-21) \mathrm{ppm}$ in $\mathrm{SFt}_{3}$ and $\mathrm{Rk}_{1}$, and $\delta_{\mathrm{C}} 140.5(\mathrm{C}-20)$ and $123.5(\mathrm{C}-22) \mathrm{ppm}$ in $\mathrm{Rg}_{5}$, respectively.

\subsection{Antiproliferative effect of the transformation products on the cancer cell lines}

The antiproliferative activities of the PNL extract before and after microwave processing, and transformation products 1-6 were evaluated in two human cancer cell lines (HeLa and A549) by the MTT assay. The results are expressed as the $\mathrm{IC}_{50}$ of the half maximal inhibitory concentration against cancer cells. As shown in Table 3, the extract of stems and leaves of P. notoginseng (EL) had no inhibitory effect on human cervical cancer (Hela) or human lung cancer (A549), and no IC $_{50}$ values were obtained. After the EL microwave treatment, microwave processing of the MEL resulted in better inhibitory activity against Hela and A549 cells, with $\mathrm{IC}_{50}$ values of 42.97 and $50.47 \mu \mathrm{g}$ $\mathrm{mL}^{-1}$, respectively. Microwave processing of ginseng enhances its anticancer activity due to the increased content of the ginsenosides $\mathrm{Rg}_{3}, \mathrm{Rg}_{5}$, and $\mathrm{Rk}_{1} \cdot{ }^{13,14}$ Further research showed that the transformation products of ginsenosides $20(S)-\mathrm{Rg}_{3}, 20(R)$ $\mathrm{Rg}_{3}, \mathrm{Rk}_{1}, \mathrm{Rg}_{5}$, and $20(S)-\mathrm{Rh}_{2}$ and notoginsenoside $\mathrm{SFt}_{3}$ in the MEL actively inhibited the Hela and A549 cells. Among compounds 1-6, ginsenoside $20(S)-\mathrm{Rh}_{2}(\mathbf{6})$ displayed lower $\mathrm{IC}_{50}$ values ( 8.23 and $12.45 \mu \mathrm{M})$ than cisplatin $(8.43$ and $16.85 \mu \mathrm{M})$ in Hela and A549 cells, respectively. The ginsenoside $20(S)-\mathrm{Rh}_{2}$ effectively inhibits tumor cell growth and survival in both animal models and cell lines. ${ }^{29-31} 20(S)-\mathrm{Rh}_{2}$ directly binds to Annexin $\mathrm{A}_{2}$, which promotes apoptosis by inhibiting NF-kB activity and down-regulating anti-apoptosis gene expression. ${ }^{31}$ 




notoginsenoside-Fa $R 1=G l c(2-1) G \mid c(2-1) X y l ; R 2=G I c(2-I) G l c$ notoginsenoside-Fc R1=Glc(2-I)Glc(2-I)Xyl; R2=Glc(6-I)Xyl
(1) Removal of glycosyl residues at C-20

(2) Dehydration at C-20

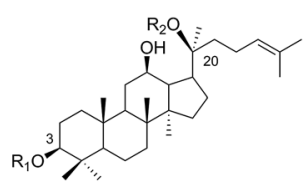

ginsenoside- $R b_{1} \quad R 1=G|c(2-I) G| c ; R 2=G \mid c(6-\mid) G l c$ ginsenoside- $R b_{2} \quad R 1=G|c(2-1) G| c ; R 2=G \mid c(6-1) A r a p$ ginsenoside- $R b_{3} \quad R 1=G|c(2-1) G| c ; R 2=G|c(6-1) X y|$ ginsenoside-Rd $R 1=G l c(2-1) G \mid c ; R 2=G l c$

ginsenoside-Rc $\quad R 1=G l c(2-I) G \mid c ; R 2=G l c(6-1) A r a f$
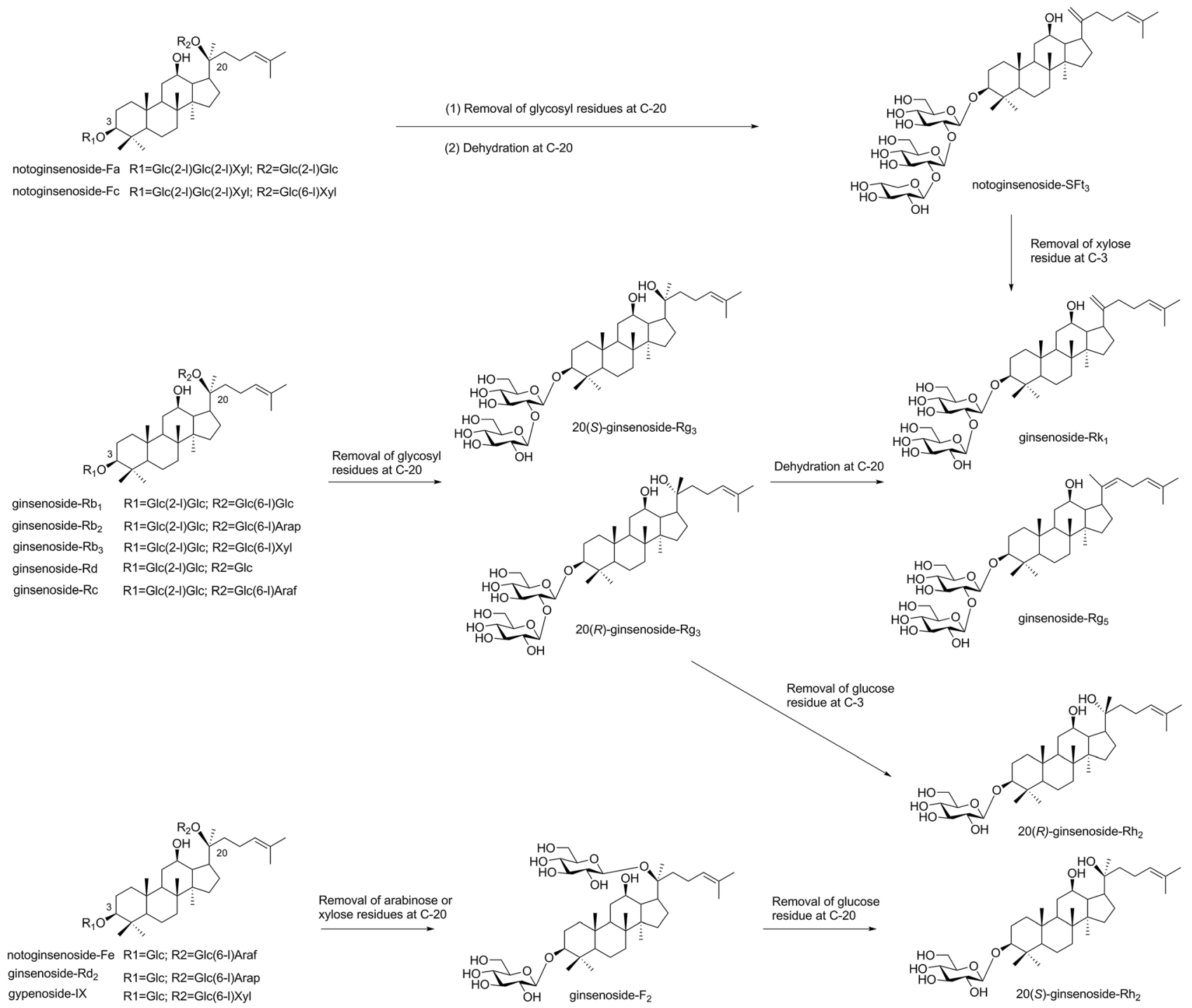

Fig. 7 Proposed transformation pathway of saponins in PNL by the microwave processing.

Table 3 Antiproliferative effect of the extracts ( $E L$ and $M E L, \mu \mathrm{gL}^{-1}$ ) and compounds 1-6 $(\mu \mathrm{M})$ in human cancer cell lines ${ }^{a}$

\begin{tabular}{lcr}
\hline & \multicolumn{2}{c}{ IC $_{50}$ values } \\
\cline { 2 - 3 } Compounds & Hela & A549 \\
\hline 20(S)-Ginsenoside $\mathrm{Rg}_{3}(\mathbf{1})$ & 23.96 & 20.87 \\
$20(R)$-Ginsenoside $\mathrm{Rg}_{3}(\mathbf{2})$ & 24.87 & 28.47 \\
Notoginsenoside $\mathrm{SFt}_{3}(\mathbf{3})$ & 19.67 & 21.48 \\
Ginsenoside $\mathrm{Rk}_{1}(\mathbf{4})$ & 11.97 & 9.52 \\
Ginsenoside $\mathrm{Rg}_{5}(\mathbf{5})$ & 42.96 & 45.24 \\
20(S)-Ginsenoside $\mathrm{Rh}_{2}(\mathbf{6})$ & 8.23 & 12.45 \\
Cisplatin & 8.43 & 16.85 \\
MEL & 42.97 & 50.47 \\
EL & $>200$ & $>200$
\end{tabular}

${ }^{a}$ Hela: human cervical cancer. A549: human lung cancer. $\mathrm{IC}_{50}$ : concentration that inhibits $50 \%$ of cell growth. EL: extract of stems and leaves of $P$. notoginseng. MEL: microwave processing extract of stems and leaves of $P$. notoginseng.
To confirm the antiproliferative effect of the transformation products, the morphologies in Hela and A549 cells treated with ginsenoside $20(S)-\mathrm{Rh}_{2}$ was observed under a microscope (Fig. 8). In the treatment of Hela and A549 cells, $20(S)-\mathrm{Rh}_{2}$ at the concentration of $1 \mu \mathrm{M}$ had no significant effect on cell morphology. While increasing concentration to 10 or $25 \mu \mathrm{M}$, $20(S)-\mathrm{Rh}_{2}$ induced cell body shrinkage and death in Hela and A549 cells.

\subsection{The anticoagulant effect of the transformation products in vitro}

According to previous reports, different parts of $P$. notoginseng have anticoagulant activity, and the leaves possess stronger anticoagulant activity among them. ${ }^{32}$ The components of the PNL changed dramatically after the microwave processing treatment (Fig. 3), which may have resulted in the change in anticoagulant activity. Therefore, the effects of the EL, MEL and transformation products 1-6 on anticoagulation were evaluated by the PT assay (Fig. 9). The PT value reflects the activity of the 
A

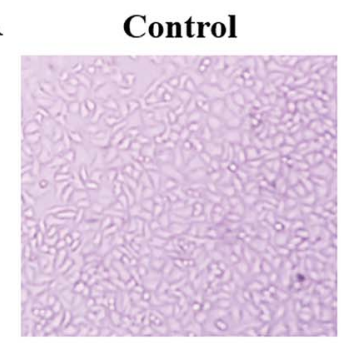

B

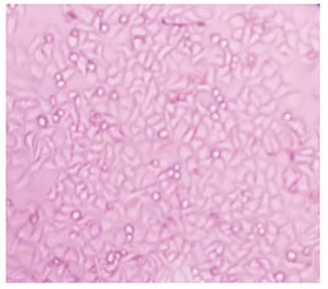

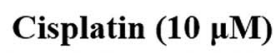
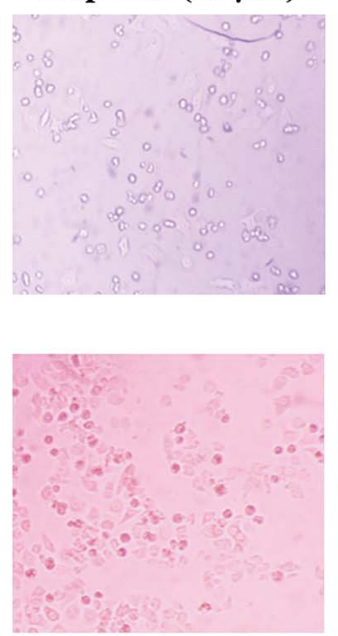

$20(S)-\mathrm{Rh}_{2}(1 \mu M)$


$20(S)-\mathrm{Rh}_{2}(10 \mu M)$

$20(S)-\mathrm{Rh}_{2}(25 \mu \mathrm{M})$

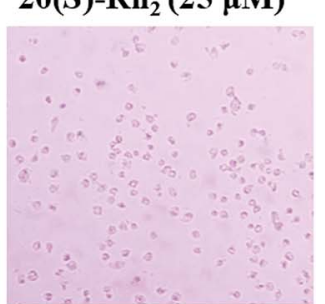

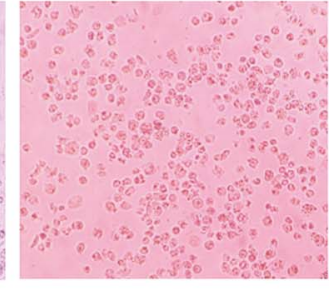

$\times 100$

Fig. 8 20(S)-Ginsenoside Rh2 inhibits cell proliferation of human cervical cancer Hela (A) and human lung cancer A549 (B). The cells were treated with 20(S)-ginsenoside Rh2 at different concentrations.

A

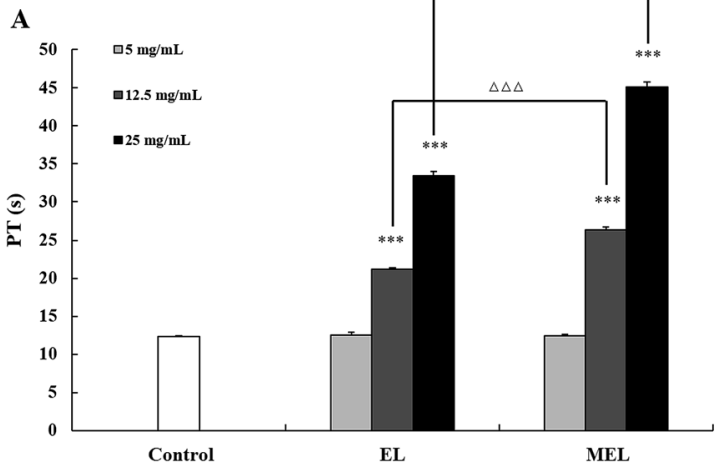

\section{B}

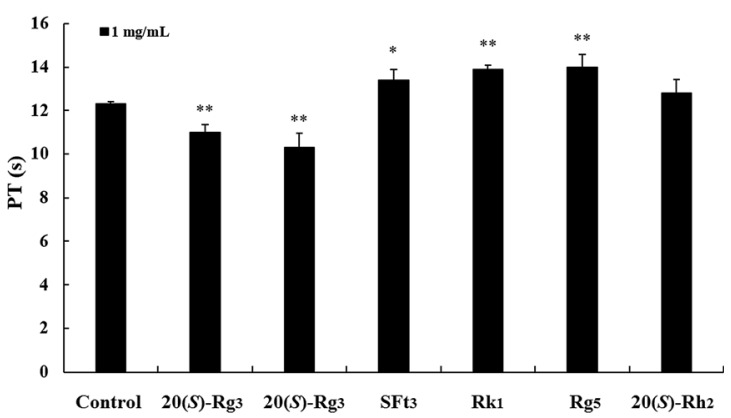

Fig. 9 Anticoagulant activities of raw and microwave processing extracts of the stems and leaves of $P$. notoginseng. (A) PT assays of the extract of stems and leaves of $P$. notoginseng (EL) and microwave processing extract of stems and leaves of $P$. notoginseng (MEL). (B) PT assays of ginsenosides 20(S)-Rg3, 20(R)-Rg3, Rk1, Rg5, 20(S)-Rh2, and notoginsenoside SFt3. Data are expressed as mean \pm SD $(n=3)$. Compared with the negative control group: ${ }^{*} p<0.05,{ }^{*} p<0.01, * * * p<0.001$. EL group compared with MEL group: $\Delta p<0.05, \Delta \Delta p<0.01, \Delta \Delta \Delta p<0.001$.

coagulation system in vitro. ${ }^{33}$ As shown in Fig. 9A, the EL and MEL had significantly prolonged clotting times in a concentration-dependent manner $(p<0.001)$. The anticoagulant activity of the MEL was better than that of the EL after comparing the extracts of the PNL before and after the microwave treatment. Further studies showed that the degradation products of ginsenosides $\mathrm{Rk}_{1}$ and $\mathrm{Rg}_{5}$, as well as notoginsenoside $\mathrm{SFt}_{3}$ prolonged coagulation time, while ginsenosides $20(S / R)-\mathrm{Rg}_{3}$ shortened coagulation time (Fig. 9B). The structure-activity results revealed that the hydroxy-substituted compounds at C$20\left(20(S / R)-\mathrm{Rg}_{3}\right)$ had the hemostatic activities, while $\mathrm{Rk}_{1}, \mathrm{SFt}_{3}$ and $\mathrm{Rg}_{5}$ with double bond at $\Delta 20(21)$ or $\Delta 20(22)$ played the anticoagulant activities. The anticoagulant activity of the MEL increased due to increases in the concentrations of $\mathrm{Rk}_{1}, \mathrm{Rg}_{5}$, and $\mathrm{SFt}_{3}$. This result was consistent with previous findings that ginsenosides $\mathrm{Rk}_{1}$ and $\mathrm{Rg}_{5}$ inhibit arachidonic acid-induced platelet aggregation in a dose dependent manner. ${ }^{34}$

\section{Conclusion}

In summary, microwave processing was applied to produce rare saponins in the PNL. Six transformation products of ginsenosides $20(S)-\mathrm{Rg}_{3}, 20(R)-\mathrm{Rg}_{3}, \mathrm{Rk}_{1}, \mathrm{Rg}_{5}, 20(S)-\mathrm{Rh}_{2}$, and notoginsenoside $\mathrm{SFt}_{3}$ were isolated from the MEL. Microwave power, temperature, and time affected the yields of the six transformation products. This transformation method was widely applied for producing the minor ginsenosides in stems and leaves, flowers, seeds and pedicels of $P$. notoginseng. The MEL had an obvious inhibitory effect on human cervical cancer and human lung cancer cell lines. The MEL had a stronger anticoagulant effect due to increased contents of ginsenosides $\mathrm{Rk}_{1}, \mathrm{Rg}_{5}$, and notoginsenoside $\mathrm{SFt}_{3}$. Therefore, the PNL may be a good source of anticancer and anticoagulant therapeutics after microwave processing. 


\section{Conflicts of interest}

All authors declare no conflicts of interest.

\section{Acknowledgements}

This work was supported by key special project of National Key Research and Development program (2017YFC1702506), analysis and special project on Science and Technology Program of Yunnan (2017ZF001 and 2018ZF013), and Scientific Research Foundation of Chongqing University of Arts and Sciences (R2015CH12 and R2015CH10).

\section{References}

1 Y. Qu, Y. Liu, L. Q. Huang, L. P. Guo and X. M. Cui, China J. Chin. Mater. Med., 2014, 39, 601-605.

2 J. M. Zhou, X. M. Cui, W. B. Zhang, H. C. Zeng and N. Ma, Res. Pract. Chin. Med., 2009, 23, 39-40.

3 F. Liu, N. Ma, C. W. He, Y. J. Hu, P. M. Li, W. Chen, H. X. Su and J. B. Wan, J. Ginseng Res., 2018, 42, 149-157.

4 Q. Mao, J. Yang, X. M. Cui, J. J. Li, Y. T. Qi, P. H. Zhang and Q. Wang, J. Pharm. Biomed. Anal., 2012, 59, 67-77.

5 T. Wang, R. X. Guo, G. H. Zhou, X. D. Zhou, Z. Z. Kou, F. Sui, C. Li, L. Y. Tang and Z. J. Wang, J. Ethnopharmacol., 2016, 188, 234-258.

6 M. Sun, Y. Ye, L. Xiao, X. Duan, Y. Zhang and H. Zhang, Int. J. Mol. Med., 2017, 39, 507-518.

7 M. Peng, Y. X. Yi, T. Zhang, Y. Ding and J. Le, Front. Pharmacol., 2018, 9, 188-206.

8 P. Lu, W. Su, Z. H. Miao, H. R. Niu, J. Liu and Q. L. Hua, Chin. J. Integr. Med., 2008, 14, 33-36.

9 R. F. Wang, J. Li, H. J. Hu, J. Li, Y. B. Yang, L. Yang and Z. T. Wang, J. Ginseng Res., 2018, 42, 270-276.

10 H. S. Choi, S. Y. Kim, Y. Park, E. Y. Jung and H. J. Suh, J. Ginseng Res., 2014, 38, 264-269.

11 L. Cui, S. Q. Wu, C. A. Zhao and C. R. Yin, J. Ginseng Res., 2016, 40, 366-374.

12 D. Wang, P. Y. Liao, H. T. Zhu, K. K. Chen, M. Xu, Y. J. Zhang and C. R. Yang, Food Chem., 2012, 132, 1808-1813.

13 P. Choi, J. Y. Park, T. Kim, S. H. Park, H. K. Kim, K. S. Kang and J. Ham, J. Funct. Foods, 2015, 14, 613-622.

14 J. Y. Park, P. Choi, H. K. Kim, K. S. Kang and J. Ham, J. Ginseng Res., 2016, 40, 62-67.
15 S. A. Galema, Chem. Soc. Rev., 1997, 26, 233-238.

16 F. Chemat, N. Rombaut, A. Meullemiestre, M. Turk, S. Perino, A. S. Fabiano-Tixier and M. Abert-Vian, Innovative Food Sci. Emerging Technol., 2017, 41, 357-377.

17 H. Y. Liu, J. Pan, Y. Yang, X. M. Cui and Y. Qu, Molecules, 2018, 23, 1243.

18 H. Yang, J. Y. Kim, S. O. Kim, Y. H. Yoo and S. H. Sung, J. Ginseng Res., 2014, 38, 194-202.

19 Q. Liu, J. J. Lv, M. Xu, D. Wang, H. T. Zhu, C. R. Yang and Y. J. Zhang, Nat. Prod. Bioprospect., 2011, 1, 124-128.

20 J. Meng and W. W. Duan, West China J. Pharm. Sci., 2017, 32, 284-286.

21 H. Z. Li, Y. J. Zhang and C. R. Yang, Nat. Prod. Res. Dev., 2006, 18, 549-554.

22 Q. K. Shen, Z. A. Chen, H. J. Zhang, J. L. Li, C. F. Liu, G. H. Gong and Z. S. Quan, J. Enzyme Inhib. Med. Chem., 2018, 33, 324-333.

23 C. Geng, J. Y. Yin, X. H. Yu, Y. X. Yang, J. Y. Liu, D. D. Sun, F. B. Chen, Z. L. Wei, Q. Meng and J. H. Liu, Rapid Commun. Mass Spectrom., 2015, 29, 283-294.

24 X. R. Zhang, J. Zhang, W. Li, L. Liu, B. S. Sun, Z. H. Guo, C. H. Shi and Y. Q. Zhao, PLoS One, 2014, 9, e94962.

25 A. Gowen, N. Abu-Ghannam, J. Frias and J. Oliveira, Trends Food Sci. Technol., 2006, 17, 177-183.

26 M. Jacotet-Navarro, N. Rombaut, S. Deslis, A. S. FabianoTixier, F. X. Pierre, A. Bily and F. Chemat, Green Chem., 2016, 18, 3106-3115.

27 J. Shao, Y. Yang and Q. Zhong, Polym. Degrad. Stab., 2003, 82, 395-398.

28 K. S. Kang, N. Yamabe, H. Y. Kim, T. Okamoto, Y. Sei and T. Yokozawa, J. Ethnopharmacol., 2007, 113, 225-232.

29 Q. Shi, J. Li, Z. Feng, L. Zhao, L. Luo, Z. You, D. Li, J. Xia, G. Zou and D. Chen, Mol. Med. Rep., 2014, 10, 1779-1785.

30 B. Li, J. Zhao, C. Z. Wang, J. Searle, T. C. He, C. S. Yuan and W. Du, Cancer Lett., 2011, 301, 185-192.

31 Y. S. Wang, Y. J. Lin, H. Li, Y. Li, Z. G. Song and Y. H. Jin, Sci. Rep., 2017, 7, 12408-12420.

32 Y. L. Zhao, K. Fan, Y. Liu, Z. S. Liang and R. L. Han, Acta Bot. Boreali-Occident. Sin., 2013, 33, 626-631.

33 C. T. Li, H. B. Wang and B. J. Xu, Pharm. Biol., 2013, 51, 10771080.

34 J. G. Lee, Y. Y. Lee, S. Y. Kim, J. S. Pyo, H. S. Yun-Choi and J. H. Park, Die Pharmazie, 2009, 64, 602-604. 